\title{
Influence of Selected Reproductive Factors and Smoking on Age at Menopause
}

\author{
B Rumianowski, I Rotter, A Brodowska, G Adler, J Kowalski, B Karakiewicz, M Laszczyńska
}

Gesundheitswesen 2015 Jan 26 (Epub ahead of print). DOI: 10.1055/s-0034-1396847

\begin{abstract}
Introduction: Early menopause may be associated with serious health risks resulting from, e.g. decreased estrogen levels. This may occur despite hormone replacement therapy.

Aim: The aim of this study was the determination of the effect of selected reproductive factors and smoking on age at the onset of menopause in women from Szczecin and surrounding areas.

Materials and methods: Three hundred and five women after natural menopause were asked to complete a questionnaire, and blood samples were collected from them to test for the levels of follicle stimulating hormone (FSH) andoestradiol (E2).

Results: Smoking women experienced menopause on average more than a year earlier than non-smokers, but this difference was not statistically significant. There was no statistically significant effect of age at menarche or first birth on age at the last menstrual period.

Conclusion: Age at menarche and first birth were not related to age at menopause. In smoking women, menopause occurred earlier but the difference was not statistically significant.
\end{abstract}

\section{Female-specific Factors for Ischemic Heart Disease: Across the Reproductive Lifespan}

C Shufelt, T Waldman, E Wang, CN Merz

Curr Atheroscler Rep 2015;17(2):481. DOI: 10.1007/S 11883-014-0481-6

\begin{abstract}
Ischemic heart disease (IHD) is the number one health threat to women in the USA. While significant advances in femalespecific symptoms and pathophysiology have begun to improve mortality rates, a closer look at risk factors across a woman's lifespan needs to be explored. This review targets three time frames: premenopause, pregnancy and postmenopause. During premenopause, menstrual cycle patterns and estrogen status provide information for IHD risk. Pregnancy conditions provide another window of time that potentially contributes to future cardiovascular risk. Lastly, there is a rise in IHD events and mortality after menopause. Research continues to decipher the impact of estrogen decline at this stage and the effect of menopause hormone therapy as they relate to the cardiovascular health of menopausal women.
\end{abstract}




\title{
Effect of Gabapentin for the Treatment of Burning Mouth Syndrome Comorbid with Depression in Postmenopausal Women
}

MJ Kim, HS Kim, SS Park

Korean J Psychosom Med 2014 Dec;22(2):138-142

\begin{abstract}
Burning mouth syndrome is characterized by intraoral burning sensation without any organic abnormalities. This syndrome is associated with various etiological factors, such as neuropathy, malnutrition, menopause and depression. Several medications have been tried for the treatment. Those are analgesics, hormones, anticonvulsants and antidepressants. However, optimal effective pharmacologic treatment remains still unknown. The purpose of this case study is to report the clinical effectiveness of gabapentin in the treatment of burning mouth syndrome in postmenopausal women with comorbid depression. We report two menopausal women. Antidepressants were effective for improving depressive symptoms, but it had no effects on intraoral burning sensation. Gabapentin reduced intraoral burning sensation effectively for all two patients. One patient reported 55\% reduction [a decrease from 9 to 4 on visual analog scale (VAS)], the other patient reported 35\% reduction (from 8-5) of the intraoral burning sensation during 16 weeks. The minimal effective daily dose of gabapentin was $300 \mathrm{mg}$. This study suggests that gabapentin might be a useful, effective therapeutic option for treating burning mouth syndrome in postmenopausal women with comorbid depression. Further prospective clinical studies are needed to investigate the effectiveness of gabapentin in patients with burning mouth syndrome.
\end{abstract}

\section{Prevalence of Osteoporosis in Patients awaiting Total Hip Arthroplasty}

Vitor Rodrigues Domingues, Gustavo Constantino de Campos, Pérola Grimberg Plapler, Márcia Uchôa de Rezende Acta Ortop Bras 2015;23(1):34-37. DOI: http://dx.doi.org/10.1590/1413-785220/5230/00981

\section{ABSTRACT}

Objective: To evaluate the prevalence of osteoporosis in patients awaiting total hip arthroplasty.

Method: Twenty-nine patients diagnosed with hip osteoarthritis awaiting primary total arthroplasty of the hip answered WOMAC questionnaire, visual analog scale (VAS) and questions about habits, osteoporosis and related diseases. Bone mineral densitometry of the lumbar spine and hips and laboratory tests (complete blood count and examination of calcium metabolism) were performed. Weight and height were measured to calculate body mass index (BMI). The evaluated quantitative characteristics were compared between patients with and without osteoporosis using the Mann-Whitney tests.

Results: Thirteen men and 16 women with a mean age of 61.5 years old, WOMAC 51.4; EVA 6.4 and BMI 27.6 were evaluated. The prevalence of osteoporosis was $20.7 \%$, and $37.9 \%$ had osteopenia. Patients with osteoporosis were older than patients without osteoporosis $(p=0.006$ ). The mean bone mineral density of the femoral neck without hip osteoarthritis was lower than the affected side $(p=0.007)$. Thirty-five percent of patients did not know what osteoporosis is. Of these, $30 \%$ had osteopenia or osteoporosis.

Conclusion: Osteoarthritis and osteoporosis may coexist and the population waiting for total hip arthroplasty should be considered at risk for the presence of osteoporosis. Level of evidence III, Observational study.

Keywords: Arthroplasty, Bone density, Hip, Osteoarthritis, Osteoporosis, Replacement. 


\title{
Effect of Menopausal Status on Carotid Intima-Media Thickness and Presence of Carotid Plaque in Chinese Women Generation Population
}

Y Zhou, D Wang, X Yang, A Wang, X Gao, Y Guo, S Wu, X Zhao

Sci Rep 2015;5:8076. DOI: 10.1038/Srep 08076.

\begin{abstract}
Menopause is an important physiological stage in women's life. The potential association of menopause with carotid intimamedia thickness (CIMT) as well as with occurrence and stability of carotid plaque in Chinese female population is unclear. We conducted a population-based, cross-sectional study by recruiting 2,131 participants aged above 40 years from northeast of China. Carotid intima-media thickness, presence of carotid plaque and its stability were evaluated by carotid duplex sonography. Among the participants, 1,133 (53.2\%) were identified to be postmenopausal. After adjusting for potential confounding factors, presence of CIMT at 50th, 75th and $\geq 75$ th percentiles, carotid plaque and its unstable status were found to be significantly associated with the postmenopausal status $(\mathrm{p}<0.001)$. When matched the participants by age, postmenopausal status was still associated with a higher risk of having unstable plaque. Moreover, our data show that postmenopausal status is a risk factor for intracranial arterial stenosis when compared with premenopausal status in the univariate analysis $(\mathrm{OR}=1.314, \mathrm{p}=0.043)$, and such relationship is lost when the confounding factors are adjusted $(\mathrm{OR}=0.828, \mathrm{p}=0.225)$.
\end{abstract}

\section{CONCLUSION}

The vascular risk factors increase as the menopausal status changes. Compared with premenopausal status, postmenopausal status is associated with higher morbidity of CIMT, carotid plaque and its unstable status.

Please visit www.jsafoms.com for Instruction to Authors and Feedback Form 